\title{
Molecular simulation of curcumin loading on graphene and graphene oxide for drug delivery applications
}

\author{
Venkatesha Narayanaswamy $^{a}$, Sulaiman Alaabed ${ }^{a}$, Bashar Issa ${ }^{b}$, M-Ali AL-Akhras ${ }^{c}$ and Ihab \\ M. Obaidat ${ }^{\mathrm{d}^{*}}$
}

${ }^{a}$ Department of Geology, United Arab Emirates University, Al-Ain 15551, United Arab Emirates

${ }^{b}$ Department of Medical Diagnostic Imaging, College of Health Sciences, University of Sharjah, Sharjah, P.O. Box 27272, United Arab Emirates

${ }^{c}$ Physics Department, Jordan University of Science and Technology, Irbid, Jordan

${ }^{d}$ Department of Physics, United Arab Emirates University, Al-Ain 15551, United Arab Emirates

\begin{tabular}{l} 
C H R O N I C L E \\
\hline Article history: \\
Received August 1, 2020 \\
Received in revised form \\
November 29,2020 \\
Accepted December 14, 2020 \\
Available online \\
December 14, 2020 \\
\hline Keywords: \\
Graphene oxide \\
Drug delivery \\
Molecular dynamics \\
Monte carlo adsorption locator \\
pi-pi interaction
\end{tabular}

\section{Introduction}

Curcumin is a polyphenol derived from the curcuma longa plant, commonly known as turmeric. Curcumin has been extensively used in ayurveda medicine for centuries, as it is nontoxic and has a variety of therapeutic properties. ${ }^{1,2}$ More recently curcumin has been found to possess anti-cancer activities through its effect on biological pathways involved in mutagenesis, oncogene expression, cell cycle regulation, apoptosis, tumor genesis and metastasis. ${ }^{1,3}$ The multi-targeted property of curcumin is used to perform a wide spectrum of functions which are better than the other therapeutic drugs. ${ }^{4}$ The reported in vitro studies establish that the $50 \%$ of cancer cell growth was inhibited with concentration of 5-30 $\mu \mathrm{M} .{ }^{5}$ Designing the curcumin loading and releasing nanocarriers for in vivo drug delivery applications has been intensely investigated. ${ }^{6}$ Recently, graphene oxide (GO) based nanocarriers for drug delivery have attracted attention in the field of biomedical applications like biosensors, tissue engineering, gene and drug delivery, cancer therapy and bio imaging. ${ }^{7,8}$ GO sheets have desired advantages over inorganic quantum dots as they have low toxicity and low cost of synthesis. The * Corresponding author.

E-mail address: iobaidat@uaeu.ac.ae (I. M. Obaidat)

(C) 2021 Growing Science Ltd. All rights reserved. doi: $10.5267 /$ j.ccl.2020.12.003 
organic framework of GO sheets renders water insoluble drugs can be easily attached to them and delivered at the site of interest by changing the temperature and $\mathrm{pH}^{9}{ }^{9}$ This study involves molecular simulations and estimation of curcumin loading capacity of GO with different oxidation extent. Curcumin loading capacity of GO sheets depends on the hydroxyl and carbonyl groups of GO sheets. Curcumin loading capacity was obtained using Monte Carlo adsorption locator simulations. ${ }^{10}$ The adsorption locator helps to determine the basic interactions responsible for the adsorption of curcumin molecule on to the GO sheet. An integrated representation of the mean binding energy for the degree of binding between curcumin molecules and GO sheets were obtained using adsorption locator calculations. To understand the binding forces instantaneous interaction energy, deformation energy and instantaneous binding energy were obtained using MC simulations. The drug loading ability of the sheets has shown dependency on oxidation extent. Molecular dynamic simulations were conducted under neutral $\mathrm{pH}$ with NPT ensemble. The bare graphene sheets are hydrophobic in nature which prevents their use as drug delivery agent in aqueous media. ${ }^{11}$ The water compatibility of graphene is further studied by PEGylation of graphene framework. The dynamic behavior of adsorption of curcumin on to the GO sheet was studied for GO with two different oxidation extents (GO1 and GO2) using molecular dynamic simulations. MD simulations studies reveal the dynamic equilibrium states between curcumin and GO sheets. The formulation methods for drug loading on to the graphene also involves the attachment of polyethylene glycol (PEG) molecules to the graphene sheets. ${ }^{12}$ In this study, curcumin loading on the PEG coated graphene, GO1, and GO2 were studied using MC simulations. Radial distribution function calculations were obtained from MD trajectories and the equilibrium distance of separation of curcumin molecules from the GO sheets was determined. These molecular simulations will help in the optimization of the chemical nature of the nanocarriers for drug delivery, which can be manipulated during the synthesis process.

\section{Computational details}

The molecular structures of the graphene and GO were built using Accelrys Materials Studio (v7.0) software. GO1 and GO2 were built with size of the GO sheet of $20 \times 20 \AA^{2}$. Highly oxidized GO was denoted as GO1 and the least oxidized sheet as GO2. GO1 and GO2 were created by randomly adding hydroxyl and epoxied groups on to the graphene sheet. GO1 and $\mathrm{GO} 2$ have oxygen to carbon $(\mathrm{O} / \mathrm{C})$ ratios of 3.0 and 8.1, respectively. The PDB of the curcumin molecule was downloaded from PubChem (Compound CID: 969516, MF: $\mathrm{C}_{21} \mathrm{H}_{20} \mathrm{O}_{6}$, MW: $368.4 \mathrm{~g} / \mathrm{mol}$ ). The geometry and energy of the molecules were optimized using forcite module by smart algorithm and COMPASSII force field. ${ }^{13}$ The optimized structures of the graphene, GO1 and GO2 sheets, and curcumin are shown in Fig. 1.

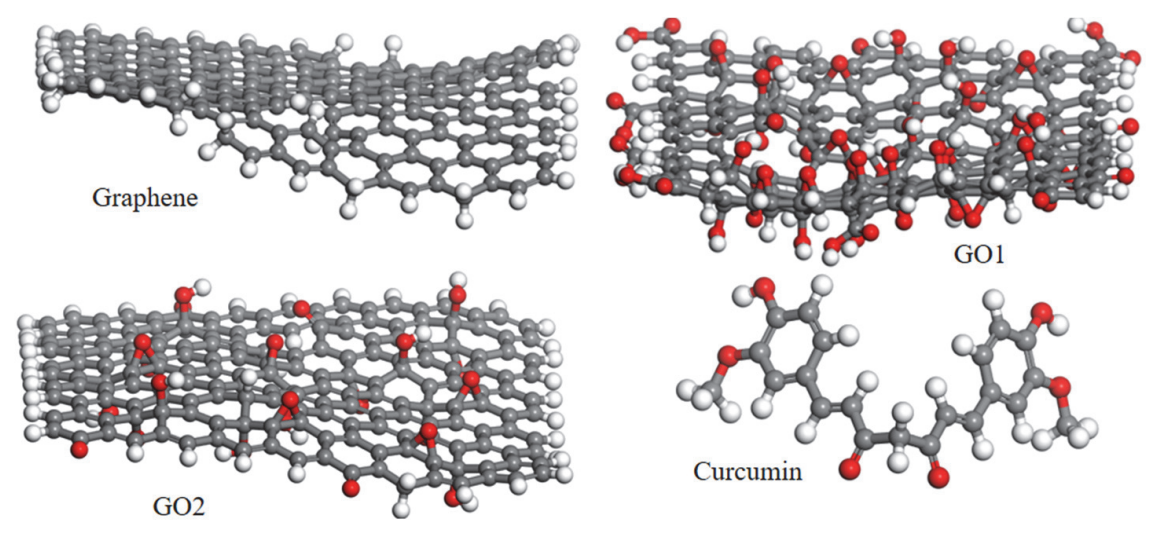

Fig. 1. Geometry and energy optimized structures (COMPASSII force field) of graphene, GO1 and GO2 sheets, and curcumin (Gray-C atoms, White-H atoms, Red-O atoms). 


\section{1 Monte Carlo adsorption locator simulations}

Adsorption locator studies were performed to determine the quantitative loading of curcumin on the graphene and GO sheets. Energy and geometry optimized curcumin were adsorbed on GO sheets using adsorption locator in material studio using COMPASSII force field. The electrostatic and Vander Waals forces of interactions were treated with Ewald and group of the atoms, respectively. ${ }^{14}$

\subsection{Molecular dynamics}

MD simulation studies were performed using forcite module in Materials studio (V7.0). MD simulations for the loading of curcumin on to the GO sheets with different oxidation extents were carried out using COMPASSII force field. The cubic simulation box with cell parameter of $33.8 \AA$ was built using amorphous cell generator by adding 5 curcumin molecules, 1 GO sheet and 1000 water molecules. The generated simulation box was energy and geometry optimized using COMPASSII force field. The simulation box temperature was maintained at $300 \mathrm{~K}$ using Nose-Hoover thermostat and pressure by Berendsen-Barostat. The short-range electrostatic interaction cut-off is set at $12.5 \AA$ using Particle-particle/particle-mesh (PPPM) ${ }^{15}$ The MD simulations for loading curcumin on to the GO sheet were studied under neutral $\mathrm{pH}$ with NPT ensemble, and the trajectory information is saved every 5 ps interval of time.

\section{Results and discussions}

\subsection{Adsorption locator studies}

The adsorption locator for curcumin molecule helps in determining the quantitative amount of curcumin drug loading on to the graphene and GO sheets. MC simulation is a configurational method, which helps in exploring the adsorption sites for the substrate molecule. Configuration search algorithm is used in $\mathrm{N}$ dimensional phase space to determine the low energy configuration. Curcumin loading capacity on to graphene, GO1, and GO2 sheets is determined and one of the configurations obtained for each substrate are shown in Fig. 2.

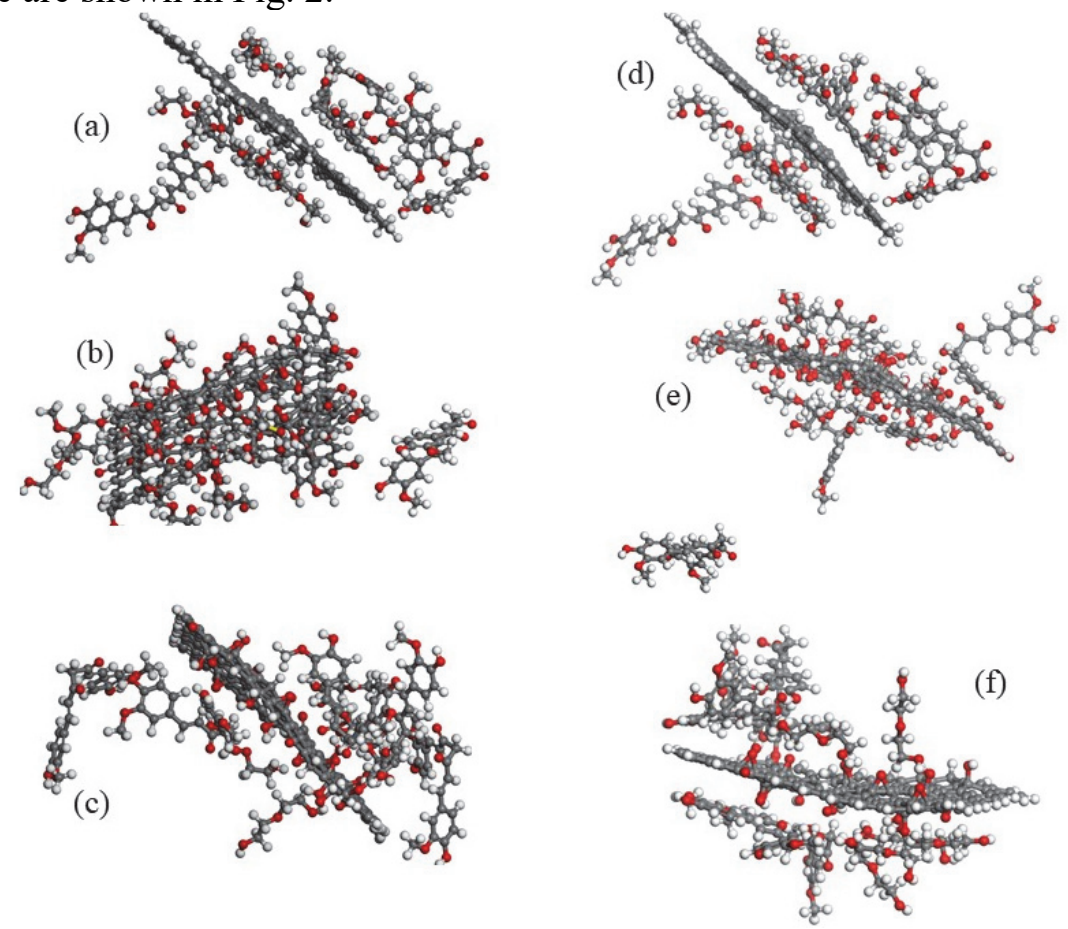

Fig. 2. One of the configurations of (a) G/curcumin, (b) GO1/curcumin, (c) GO2/curcumin, (d) GPEG/curcumin, (e) GO1-PEG/curcumin, and (f) GO2-PEG/curcumin systems obtained by adsorption locator using Monte Carlo Method 
The curcumin molecule has a bent geometry with two phenolic groups and the phenolic ring interacts with the graphene aromatic ring. In all the configurations, the phenolic ring was parallel attached to the GO sheet. From Fig. 2 it is clear that the amount of curcumin loading decreases with increasing oxidation groups. The energy distribution of adsorption loading on the graphene and GO sheets is shown in Fig. 3. The adsorption and configurational energies are listed in Table 1. From the energy calculations it is evident that hydrogen-terminated graphene has highest curcumin loading capacity. The substrate energy during calculations was set to zero and the adsorption energy was calculated using the equation 1. Total energy of the configurations obtained includes energies of adsorbate components, deformation energy upon adsorption, and rigid adsorption energy. Table 1 shows $\mathrm{dEad} / \mathrm{dNi}$, which is the energy of $\mathrm{GO} /$ Curcumin configurations when the adsorbed molecule was released. From Fig. 2, it is evident that the curcumin molecule prefers aromatic framework of the graphene over the oxidation groups of GO. This is because of the pi-pi interaction, which stabilizes the adsorption of curcumin on $\mathrm{GO}$ sheet under neutral $\mathrm{pH}$. To understand the effect of functionalization of G, GO1, and GO2 with PEG on curcumin loading on graphene framework MC simulations were employed. The MC confiscations obtained for PEG functionalized graphene frameworks are shown in Fig. 2(d, e, and f). The energy profiles are shown in Fig. 3(d, e, and f). The adsorption data shows that PEGylation of graphene framework does not improve the curcumin loading.
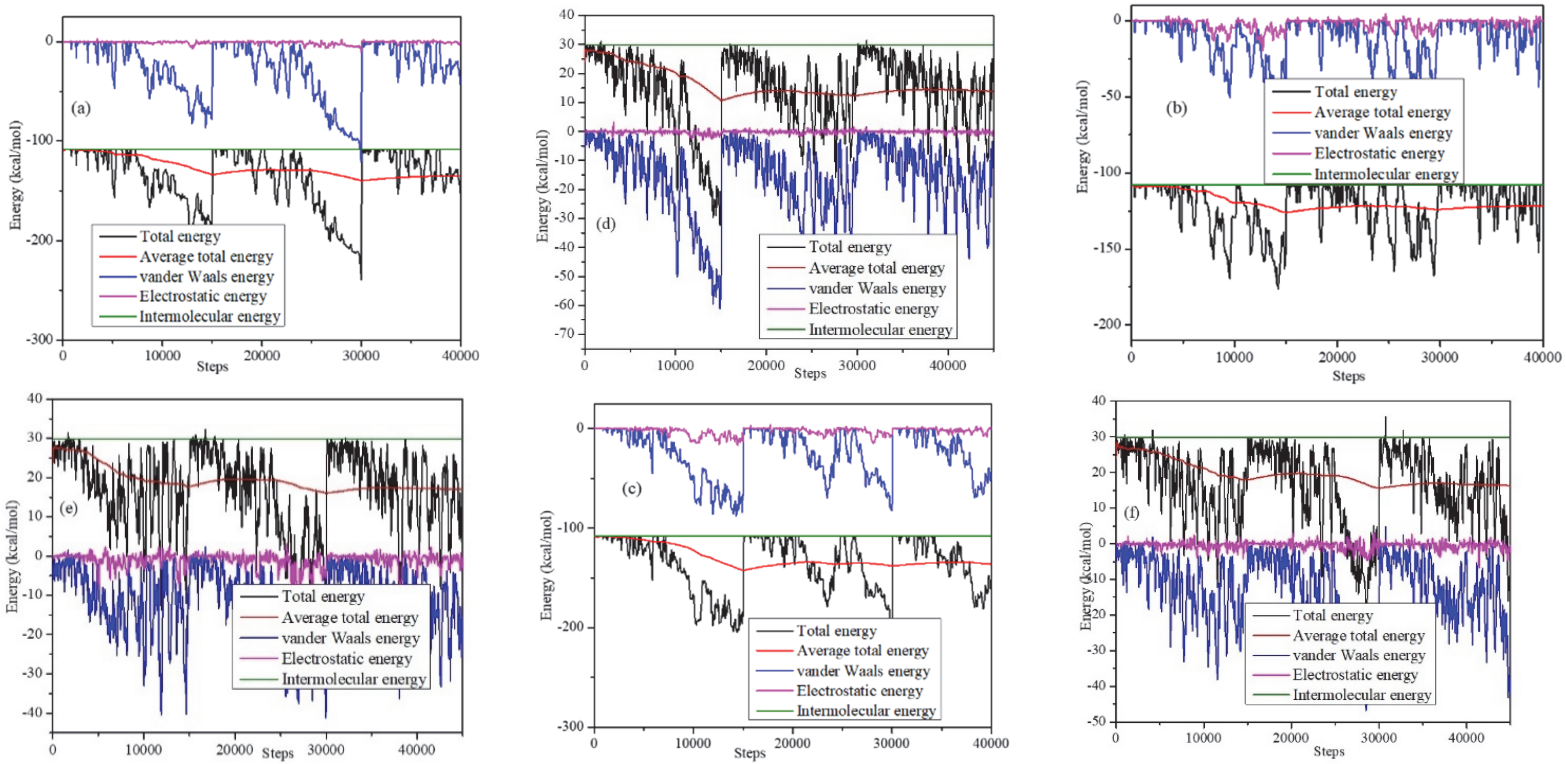

Fig. 3. Energy distribution of adsorption locator for (a) G/curcumin, (b) GO1/curcumin, (c) GO2/curcumin, (d) G-PEG/curcumin, (e) GO1-PEG/curcumin, and (f) GO2-PEG/curcumin systems.

Table 1. Energies calculated using adsorption locator simulations. Energy of the most stable graphene/GO1,2-Curcumin configurations. (GO1 has more hydroxyl and epoxide functional groups compared to $\mathrm{GO} 2)$.

\begin{tabular}{cccccc}
\hline Structures & Total energy & Adsorption energy & Rigid adsorption energy & Deformation energy & cur : dEad/dNi \\
\hline Substrate & 0 & & & & \\
Curcumin & -21.6323 & & & & \\
G & -271.8556 & -163.6936 & -169.2822 & 5.5886 & -33.4617 \\
GO1 & -204.3149 & -96.1529 & -100.2700 & 4.1171 & -23.1342 \\
GO2 & -255.7880 & -147.6260 & -153.6490 & 6.0233 & -22.0816 \\
G-PEG & -44.6186 & -74.5680 & -75.8554 & 1.2873 & -10.2506 \\
GO1-PEG & -35.9312 & -65.8806 & -67.1913 & 1.3107 & -3.0020 \\
GO2-PEG & -42.8358 & -72.7852 & -74.0453 & 1.2601 & -8.3355 \\
\hline
\end{tabular}

\subsection{Molecular dynamics simulations}

MD simulations of GO1, GO2/Curcumin were conducted in aqueous solution under neutral $\mathrm{pH}$ using molecular dynamics using forcite module. The simulation box obtained from amorphous cell was 
energy and geometry optimized. The total energy fluctuation during the MD simulations are provided in Fig. 4 (a, b). To evaluate the equilibrium adsorption distance between curcumin molecules and GO1 and $\mathrm{GO} 2$ sheets, radial distribution function (RDF) calculations were analyzed. The RDF of the curcumin molecule adsorption on GO sheets is shown in Fig. 4(c).

$\mathrm{RDF}$ is an important estimation of probable distance of separation of dyes from the GO surface. RDF is calculated using Eq. (1). ${ }^{16}$

$$
\mathrm{g}_{\mathrm{XY}}(\mathrm{r})=\frac{\rho_{\mathrm{Y}}(\mathrm{r})}{\rho_{\mathrm{Y}}}
$$

$\rho_{\mathrm{Y}}(\mathrm{r})$ : Average density of molecule $\mathrm{Y}$ at a distance $\mathrm{r}$, around the molecule $\mathrm{X}$.

$\rho_{\mathrm{Y}}$ : Density of the molecule $B$ averaged over all spheres around the molecule $\mathrm{X}$ until $\mathrm{r}_{\max }$.

The distances of separation curcumin molecules which are attached on GO1 and GO2 sheets are 4.38 and $4.4 \AA$ which were obtained from the radial distribution curves shown in Fig. 4(c).
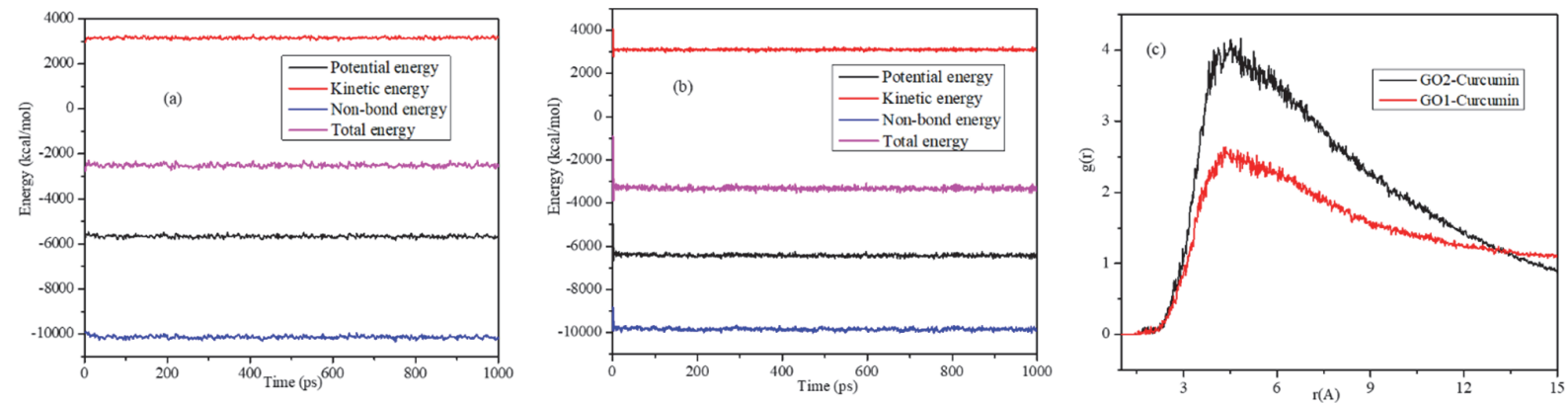

Fig. 4. Total energy fluctuations during Molecular dynamics simulations for $1000 \mathrm{ps}$ of the (a) Curcumin/GO1, (b) Curcumin /GO2, and (c) Radial Distribution Functions between curcumin and

GO
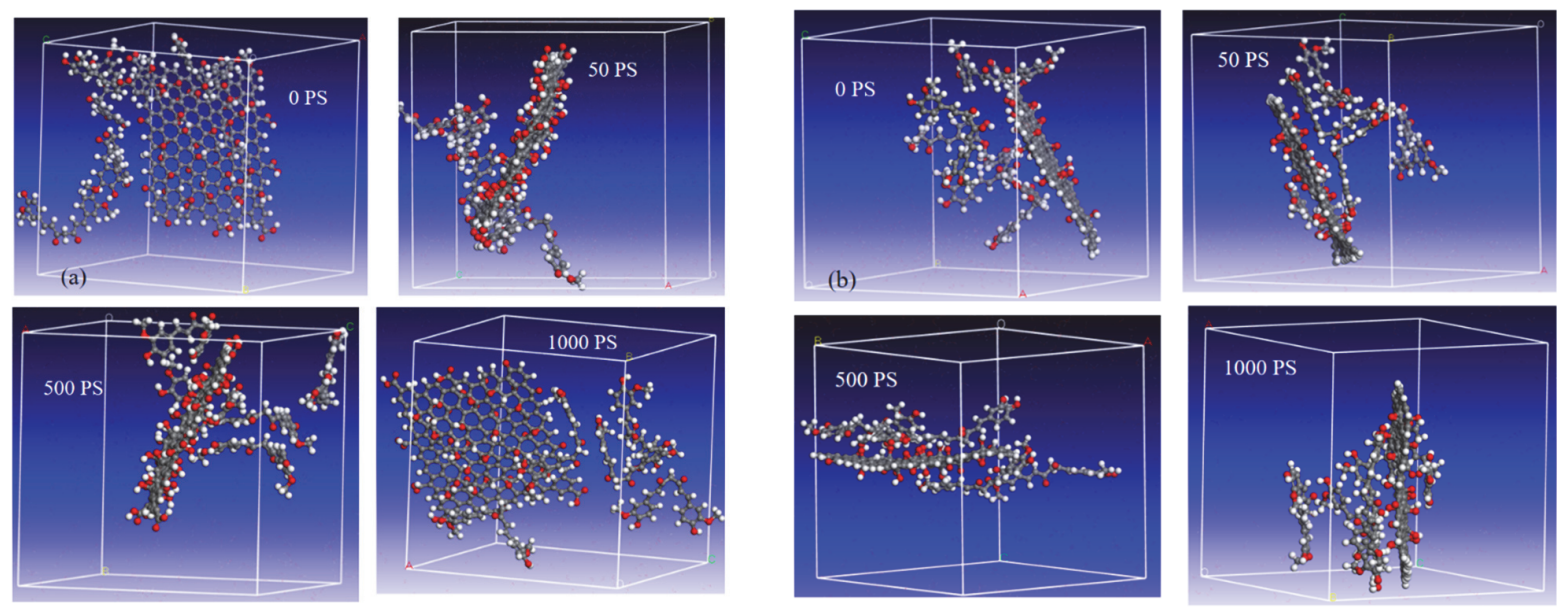

Fig. 5. Snapshots of MD simulations for the (a) Curcumin/GO1 and (b) Curcumin/GO2 systems.

The snapshots at 0, 50, 500, and 1000 ps simulation times are shown in Fig. 5. At 0 ps the molecules were randomly arranged for better visibility of the curcumin and GO sheet. The water molecules font size was set to zero. From the Fig. 5 it is clear that the solubility of curcumin molecules in water is limited and less number of molecules were attached to GO1 framework compared to GO2 at 1000 ps. The orientation of attachment of curcumin molecules on GO sheets shows that the preferred interaction is $\pi-\pi$ between aromatic rings of graphene and phenolic ring of curcumin. The equilibrium distance of separation of curcumin molecule from GO sheet obtained from $\mathrm{MC}$ and $\mathrm{MD}$ simulations are 
comparable. To load the maximum amount of curcumin to form the nano-formulations, optimum oxidation of GO sheet with water compatibility is required.

\section{Conclusions}

Curcumin drug loading capacity on the graphene oxide sheets with different oxidation extents were determined using Monte Carlo adsorption locator and MD simulations. MC simulations and the adsorption energy calculations obtained for graphene, $\mathrm{GO}$ /Curcumin configurations shows that the drug loading capacity is high for the pristine graphene whereas it is the least for the highly oxidized graphene oxide sheet GO1. Molecular dynamic simulations conducted under NPT ensemble under neutral $\mathrm{pH}$ also shows that the loading of curcumin is least for the highly oxidized sheet GO1. Although, the loading capacity is different for GO1 and GO2, the equilibrium distance of curcumin from both sheets is $4.4 \AA$. The equilibrium configurations obtained after $1000 \mathrm{ps}$ simulation time shows that only two molecules were adsorbed on the GO1 sheet, whereas four curcumin molecules are adsorbed on GO2 sheet. The simulation studies presented here give insight for the nano formulation of curcumin loading and releasing agents, which can be used to synthetically design the properties of GO sheets.

\section{Acknowledgment}

This work was financially supported by UAEU Program for Advanced Research (UPAR) under Grant no.31S312.

\section{References}

1. Wilken R., Veena M.S., Wang M.B., and Srivatsan E.S. (2011) Curcumin: A review of anti-cancer properties and therapeutic activity in head and neck squamous cell carcinoma. Mol. Cancer 10 (12) $1-19$.

2. Mahmood K., Zia K.M., Zuber M., Salman M., and Anjum M.N. (2015) Recent developments in curcumin and curcumin based polymeric materials for biomedical applications: A review. Int. J. Biol. Macromol. 81, 877-890.

3. Kanai M. (2014) Therapeutic applications of curcumin for patients with pancreatic cancer. World J Gastroenterol 20 (28) 9384-9391.

4. Tomeh M.A., Hadianamrei R., and Zhao X. (2019) A Review of Curcumin and Its Derivatives as Anticancer Agents. Int J Mol Sci 20 (5) 1033.

5. Hosseini S.A., Zand H., and Cheraghpour M. (2019) The Influence of Curcumin on the Downregulation of MYC, Insulin and IGF-1 Receptors: A Possible Mechanism Underlying the Anti-Growth and Anti-Migration in Chemoresistant Colorectal Cancer Cells. Medicina 55 (4) 90.

6. Deng Y., Verron E., and Rohanizadeh R. (2016) Molecular Mechanisms of Anti-metastatic Activity of Curcumin. Anticancer Res 36 (11) 5639-5647.

7. Chung C., Kim Y.-K., Shin D., Ryoo S.R., Hong B.H., and Min D.H. (2013) Biomedical Applications of Graphene and Graphene Oxide. Acc. Chem. Res. 46 (10) 2211-2224.

8. Dasari S.T.P., McShan D., Dasmahapatra A.K., and Tchounwou P.B. (2018) A Review on Graphene-Based Nanomaterials in Biomedical Applications and Risks in Environment and Health. Nano-Micro Lett. 10 (3) 53.

9. Liu C.-C., Zhao J.J., Zhang R., Li H., Chen B., Zhang L.L., and Yang, H. (2017) Multifunctionalization of graphene and graphene oxide for controlled release and targeted delivery of anticancer drugs. Am J Transl Res 9 (12) 5197-5219.

10. Akkermans R.L.C., Spenley N.A., and Robertson S.H. (2013) Monte Carlo methods in Materials Studio. Molecular Simulation 39 (14-15) 1153-1164. Taylor \& Francis.

11. Dao T.D., Erdenedelger G., and Jeong H.M. (2014) Water-dispersible graphene designed as a Pickering stabilizer for the suspension polymerization of poly(methyl methacrylate)/graphene core-shell microsphere exhibiting ultra-low percolation threshold of electrical conductivity. Polymer 55 (18) 4709-4719. 
12. Kazempour M., Namazi H., Akbarzadeh A., and Kabiri R. (2019) Synthesis and characterization of PEG-functionalized graphene oxide as an effective $\mathrm{pH}$-sensitive drug carrier. Artificial Cells, Nanomedicine, and Biotechnology 47(1) 90-94. Taylor \& Francis.

13. Sun H., Jin Z., Yang C., Akkermans R.L.C., Robertson S.H., Spenley N.A., Miller S., and Todd S.M. (2016) COMPASS II: extended coverage for polymer and drug-like molecule databases. $J$ Mol Model 22 (2) 47.

14. Wang F., Xia W., Wang L., Wang J., Yang X., and Chen K. (2018) Grand Canonical Monte Carlo Simulations of Ethanol Conversion to Propylene Over Zeolite Catalysts. Front. Mater. 5. Frontiers.

15. Liu J., Li P., Xiao H., Zhang Y., Shi X., Lu X., and Chen X. (2015) Understanding flocculation mechanism of graphene oxide for organic dyes from water: Experimental and molecular dynamics simulation. AIP Advances 5 (11) 117151. American Institute of Physics.

16. Levine B.G., Stone J.E., and Kohlmeyer A. (2011) Fast Analysis of Molecular Dynamics Trajectories with Graphics Processing Units-Radial Distribution Function Histogramming. $J$ Comput Phys 230 (9) 3556-3569. 
(C) 2021 by the authors; licensee Growing Science, Canada. This is an open access article distributed under the terms and conditions of the Creative Commons Attribution (CC-BY) license (http://creativecommons.org/licenses/by/4.0/). 